\title{
The Role of Chinese Face in the Perpetration of Dating Partner Violence
}

Journal of Interpersonal Violence $\mathrm{XX}(\mathrm{X}) \mathrm{1}-19$

(c) The Author(s) 2011

Reprints and permission: http://www. sagepub.com/journalsPermissions.nav DOI: I0.I I77/08862605 | |423242 http://jiv.sagepub.com

\section{Ko Ling Chan' [AQ: 1]}

\begin{abstract}
This study explored the associations between the perpetration of partner violence and two types of face orientation - protective and acquisitive-in Chinese societies. Data from a convenience sample of 3,388 university students from Hong Kong, Shanghai, and Beijing were analyzed.The participants completed the Protective and Acquisitive Face Orientation (PAFO) Scale Short Form and the Revised Conflict Tactics Scale (CTS2) to measure their face orientations and experience of perpetrating violence against their dating partner.Acquisitive face orientation (AFO) was positively associated with the self-reported perpetration of physical and psychological partner violence. No significant associations were found between protective face orientation (PFO) and partner violence perpetration. Gender differences were found across all types of dating violence. City of residence, age, and SES were also associated with partner violence in specific ways. The findings gave insights on the possible mechanisms between partner violence and the concept of face to be explored in future research.
\end{abstract}

\section{Keywords}

Chinese face, dating violence, intimate partner violence, perpetration

\footnotetext{
'University of Hong Kong, Hong Kong, China
}

\section{Corresponding Author:}

Ko Ling Chan, Department of Social Work and Social Administration, The University of Hong Kong, Pokfulam, Hong Kong 852,SAR, China.

Email: eklchan@hkucc.hku.hk 
Intimate partner violence (IPV) has long been demonstrated to have serious harmful effects on its victims (e.g., Campbell \& Soeken, 1999; Lindhorst \& Oxford, 2008; Yoshihama, Horrocks, \& Kamano, 2009). To help combat IPV, researchers and scientists have focused on its various risk factors and protective factors, including personal factors such as substance abuse (Caetano, McGrath, Ramisetty-Mikler, \& Field, 2005; Golinelli, Longshore, \& Wenzel, 2009) and depressive symptoms (Lehrer, Buka, Gortmaker, \& Shrier, 2006; Sugarman, Aldarondo, \& Boney-McCoy, 1996), as well as family factors such as relationship distress (Stuart \& Holtzworth-Munroe, 2005; VivesCases, Gil-Gonzalez, \& Carrasco-Portino, 2009) and financial burden (Balmer, Pleasance, Buck, \& Walker, 2005; Chan, Brownridge, Tiwari, Fong, $\&$ Leung, 2008). Cultural factors also play a role in IPV. For example, the Latino concept of machismo, which can be defined as values and behaviors associated with masculinity, invulnerability, and bravery (Whitaker \& Reese, 2007), may encourage men to be controlling and aggressive to their wives who are believed to have the obligation of serving and being available for men. As another example, the high value placed on Confucianism, which emphasizes gender roles and hierarchy within family, among Chinese populations may increase the risk of violence on women as wives are often expected to be absolutely obedient to her family and husband (Tiwari et al., 2009). Aiming at exploring other possible factors associated with IPV in the Chinese populations, the present study focused on another important concept of the Chinese societies-"face."

\section{The Concept of "Face"}

The Chinese concept of "face," aka mientzu or mianzi, has been believed to play an important role on social interactions among Chinese populations (Ho, 1976, 1980; Hu, 1944). Face has been explained as "prestige; dignity; honor; respect; status" (Carr, 1993) and is similar to the Western concept of reputation. Although not exclusive to China (Goffman, 1955), face is more deeply embedded in the Chinese culture, where it is considered as a major principle governing social life (Eberhard, 1967; King \& Myers, 1977). Face can be gained or lost during social interactions and is related to various personal and relationship characteristics including self-esteem, social desirability, and interpersonal relationships (Chou, 1996). Face, on one hand, can be gained through achieving attributes that are mutually approved by oneself and the significant others, including power, wealth, and ability (Ho, 1993); and, on the other hand, can be lost when being criticized, humiliated, found failure or doing acts that are not acceptable by the social norm. Losing face has been viewed as far more serious 
implications for one's self-esteem and moral decency than gaining face (Huang, 1987). Therefore, for individuals placing high values on face, gaining face is desirable while losing face is extremely unpleasant.

Chou (1996) proposed two types of face orientation: protective face orientation (PFO) and acquisitive face orientation (AFO). Face orientation is thought to be a mediating process that influences an individual's perception, interpretations, emotional reactions, and behavioral goal setting (Lau \& Wong, 2008). Although both can influence an individual's cognition and affect, they may be related to different ways for one to selectively attend, encode, or react to social cues. Individuals with high levels of PFO are often described as having "thin face" which is easily lost, highly sensitive to others' views of them, and likely to make their greatest effort to maintain face. In other words, they emphasize "sustaining face by covering up their weaknesses and failures" (p. 25). Individuals with high PFO levels are characterized by avoidance of disapproval and disagreement and are very likely to be compliant and conforming. They also avoid taking risks to minimize the chance of being humiliated or embarrassed. In general, high-PFO individuals are modest and good at getting along with others and tend to engage in a passive yet pleasant interaction style to maintain harmonious relationships with others.

In contrast, individuals with high levels of AFO are more concerned with gaining face and tend to undertake more active work to acquire face. According to Chou (1996), high-AFO individuals are often more aggressive and active in terms of maintaining and gaining face through social interactions. They may tend to view themselves as being superior to others, show less concern for social norms and possible failures, and even adopt a "high risk, high payoff" (p. 43) approach to fulfill their face needs. Individuals with extremely high levels of AFO can have interpersonal problems because of their desire to gain face.

\section{Face and Violence}

Face orientation, which emphasizes avoiding face-losing situations which can bring into question one's moral decency and societal adequacy, often shapes the behaviors of Chinese people (Eberhard, 1967). This can be particularly obvious when immoral or social unacceptable acts are involved. Violence is one example of behaviors that may be influenced by face. Perpetration or victimization of violence, or the disclosure of violent experience to others, may lead to a loss of face. The avoidance of face-losing may serve as a barrier to the reporting of violence. Indeed, Hall and colleagues have shown in a study that the avoidance of losing face can be a protective 
factor for sexual aggression among Asian American men (Hall, Teten, DeGarmo, Sue, \& Stephens, 2005). Specifically, Asian American men with high levels of risk factors are less likely to commit sexual aggressive acts if they have higher avoidance of loss of face. The impact of face may be even greater when family member or intimate partner is involved in the violent relationship because family members or intimate partners are often involved in evaluating one's social standing or reputation. Chinese traditions have taught people not to disclose undesirable family issues to others to maintain face. In fact, in Chinese culture, women who hold traditional values may refuse to disclose victimization of partner violence as a way for preserving face of their male partners (Chan, 2006).

Based on Chou's (1996) concepts of face orientation, violence against an intimate partner may be associated with one's PFO and AFO levels. PFO may act as a buffer against the perpetration of IPV as it tends to make individuals cautious not to involve in socially undesirable behaviors or situations. PFO may also lower the tendency for one to disclose or report perpetration or victimization of IPV, which can lead to a loss of face of oneself or the partner. In contrast, AFO can increase one's aggression level and enhance one's self-perception of superiority. High AFO individuals may perpetrate IPV when they view themselves as more superior than their partners or when they adopt aggressive approaches in handling face-relevant situations in an intimate relationship.

\section{The Present Study}

With regard to the possible links between face orientations and IPV, this study aimed at exploring the associations between $\mathrm{PFO}, \mathrm{AFO}$, and partner violence, as well as other correlates of IPV, in Chinese populations. Specifically, three cities in China were selected: Beijing, Shanghai, and Hong Kong. Although all of them are metropolitan cities that are considered as urban and developed in China, they do own specific characteristics that differentiate themselves from others. For example, Hong Kong, a former British colony, is now a special administrative region where the economic, political, legal, and educational systems are different from other cities in China. Most Chinese people in Hong Kong have been exposed to Western culture for many years, especially before the sovereignty of which was returned to China in 1997. Shanghai, however, has long been a centre of commerce, finance, technology, and culture in China. Being located in coastal region, Shanghai was one of the few cities in China open to foreign traders and bankers in the past, which have led to greater exposure to Western cultures among Shanghai people. Indeed, when compared with people in Beijing which has long been the capital of 
China and less exposed to other cultures, Shanghai people are less traditional and dominant in homes (Chan, 2007).

Considering the potential socioeconomic and cultural differences between the studied cities, the patterns of partner violence perpetration may be different. Therefore, based on existing literature, it was hypothesized that (a) PFO would be negatively associated with the reporting of IPV perpetration; (b) AFO would be positively associated with IPV perpetration; and (c) IPV perpetration would be different among research sites. In addition, three other sociodemographic factors, which have been suggested to be related to IPV perpetration, were investigated: gender (Chan \& Straus, 2008; Chan, Straus, Brownridge, Tiwari, \& Leung, 2008; Straus, 2004b), age (Goodwin, Gazmararian, Johnson, Gilbert, \& Saltzman, 2000; Hedin, 2000; Martin, Mackie, Kupper, Buescher, \& Moracco, 2001; Muhajarine \& D’Arcy, 1999; Tang, 1999), and socioeconomic status (Bates, Schuler, Islam, \& Islam, 2004; Cunradi, Caetano, \& Schafer, 2002).

\section{Method}

\section{Sample and Sampling}

This study used data from the International Dating Violence Study, an international cross-sectional study of university students that investigated the prevalence and characteristics of dating partner violence, as well as its demographic and psychological correlates (Straus, 2004b). In this study, we used the data collected from university students who were residing in Hong Kong, Beijing, or Shanghai and had identified themselves as being or having been in an intimate relationship by the time of the survey study.

The study and its procedures were approved by the ethics committee of the University of Hong Kong. The participants in the study were a convenience sample of 3,388 university students from Hong Kong $(n=1,001)$, Beijing $(n=$ $1,481)$, and Shanghai $(n=906)$, representing a response rate of more than $90 \%$. Participants were recruited predominantly through social studies courses offered by 2 universities in Hong Kong, 10 universities in Beijing, and 4 universities in Shanghai. Students were told both verbally and literally that their participation in the study was voluntary and confidential. The study investigators obtained the students' written consent to participate, after which the participants completed an anonymous self-response questionnaire on dating violence and related demographic and psychological characteristics. To ensure privacy and to confidentiality, participants were allowed to complete the questionnaire anywhere they felt quiet, safe, and private. 
Table 1 shows the demographic details of the sample. Overall, the sample comprised $70 \%$ female and $30 \%$ male, with a modal age of 19 to 21 years $(75.6 \%)$. About $24.0 \%$ were or had been in a relationship of more than 2 years, and $26.5 \%$ had had sex with their current or most recent partner. Significant gender differences were found in respondents' residence city, year of study, age, status and length of the referred relationship, cohabitation status, and acquisitive face orientation.

\section{Measures}

Partner violence. Dating partner violence was measured using the Chinese translation of the Revised Conflicted Tactics Scale (CTS2; Straus, Hamby, BoneyMcCoy, \& Sugarman, 1996). In this study, the CTS2 assessed the perpetration of physical, sexual, and psychological partner violence over lifetime and during the year preceding the survey study. Three subscales were used to measure the three types of violence (i.e., physical, sexual, and psychological), and each type of violence was categorized as minor or severe. Minor physical violence included slapping, grabbing, pushing, shoving, twisting a partner's arm or hair, or throwing something at a partner, whereas severe physical violence included kicking, burning/scalding, beating up, punching, choking, hitting a partner with an object, slamming a partner against a wall, or attacking a partner with a knife or gun. Minor sexual abuse included pressuring a partner without the use or threat of physical force to have sex or to not use a condom; while severe sexual abuse included using or threatening to use physical force or a weapon to have sex. Minor psychological aggression included shouting at partner or doing something to spite partner, whereas severe psychological aggression included destroying something of partner's or threatening to hit or throw something. The response scale was dichotomous in this study: Reporting the perpetration of any of the above acts would score " 1 " for the subscale or otherwise " 0 ." The CTS2 was demonstrated to have cross-cultural validity and reliability (Straus, 2004a). In this study, the Cronbach's alpha coefficients of the CTS2 subscales ranged from .79 to .92 , indicating a satisfactory to good reliability. Participants were instructed to refer to their most recent intimate relationship when answering the CTS2. The concept of an "intimate relationship" was defined by the participants and not restricted by the study investigators.

Face. The Protective and Acquisitive Face Orientation (PAFO) Scale Short Form, which was modified from the original 50-item PAFO Scale (Chou, 1996), was used to measure the general trend in the protective and acquisitive motives of people in face-relevant situations (Wang, 2002). Two 10-item 
Table I. Demographic Characteristics of Participants

\begin{tabular}{|c|c|c|c|c|}
\hline \multirow[b]{2}{*}{ Characteristics } & \multicolumn{3}{|c|}{ Percentage (\%) } & \multirow[b]{2}{*}{$\chi^{2} / t$} \\
\hline & $\begin{array}{c}\text { Total } \\
(N=3,388)\end{array}$ & $\begin{array}{c}\text { Male } \\
(n=1,147)\end{array}$ & $\begin{array}{c}\text { Female } \\
(n=2,24 I)\end{array}$ & \\
\hline City of residence & & & & $0.000 * * *$ \\
\hline Shanghai & 26.7 & 34.4 & 22.8 & \\
\hline Beijing & 43.7 & 40.1 & 45.6 & \\
\hline Hong Kong & 29.5 & 25.5 & 31.6 & \\
\hline Year in school & & & & $0.000^{* * * *}$ \\
\hline Freshman & 44.8 & 39.3 & 47.7 & \\
\hline Sophomore & 27.7 & 26.8 & 28.2 & \\
\hline Junior & 17.6 & 19.9 & 16.4 & \\
\hline Senior & 9.8 & 14.0 & 7.7 & \\
\hline Age & & & & $0.000 * * *$ \\
\hline$\leq 18$ & 4.3 & 3.3 & 4.8 & \\
\hline$|9-2|$ & 75.6 & 70.4 & 78.3 & \\
\hline $22-24$ & 15.2 & 18.8 & 13.3 & \\
\hline$\geq 25$ & 4.9 & 7.4 & 3.6 & \\
\hline Socioeconomic status $(M, S D)$ & $(8.48,3.76)$ & $(8.37,3.82)$ & $(8.54,3.73)$ & 0.218 \\
\hline Parents' marital status & & & & 0.598 \\
\hline Married or cohabiting & 91.3 & 91.5 & 91.1 & \\
\hline Separated or divorced & 5.3 & 5.5 & 5.2 & \\
\hline Deceased & 3.4 & 3.0 & 3.6 & \\
\hline Relationship status & & & & $0.004^{* *}$ \\
\hline Previous & 21.3 & 24.6 & 19.6 & \\
\hline Current, I month or more & 36.5 & 35.2 & 37.2 & \\
\hline Current, less than I month & 42.2 & 40.3 & 43.2 & \\
\hline Relationship length & & & & $0.007^{* *}$ \\
\hline Less than 2 years & 76.0 & 79.5 & 74.1 & \\
\hline 2 years or more & 24.0 & 20.5 & 25.9 & \\
\hline Cohabitation status & & & & $0.000 * * *$ \\
\hline Alone & 4.0 & 6.8 & 2.5 & \\
\hline With partner & 3.1 & 4.5 & 2.4 & \\
\hline With roommate, nonpartner & 61.1 & 64.8 & 59.1 & \\
\hline With parent(s) & 29.5 & 20.9 & 34.2 & \\
\hline Others & 2.2 & 3.0 & 1.8 & \\
\hline Had sex with partner & & & & 0.687 \\
\hline Yes & 26.5 & 26.0 & 26.8 & \\
\hline No & 73.5 & 74.0 & 73.2 & \\
\hline Face orientation $(M, S D)$ & $54.99(5.49)$ & $55.25(6.30)$ & $54.87(5.06)$ & 1.540 \\
\hline Protective $(M, S D)$ & $28.10(3.45)$ & $28.10(3.75)$ & $28.10(3.30)$ & 0.006 \\
\hline Acquisitive $(M, S D)$ & $26.88(4.54)$ & $27.16(4.77)$ & $26.7(4.43)$ & $2.158^{*}$ \\
\hline
\end{tabular}

$*_{p}<.05 .{ }^{*} p<.01$. *** $p<.001$. 
subscales of the PAFO were employed: the Protective Face Orientation (P-subscale) and the Acquisitive Face Orientation (A-subscale). Sample items of the P-subscale included "being cautious or conscious of making mistakes"; "not talking much to avoid bringing attention"; and "keeping one's strengths out of the spotlight; talking conservatively or not showing off," whereas the A-subscale included items such as "presenting strengths to others"; "being happy with others' attention or admiration"; and "desire for being supported and respected." Participants were asked to rate on whether they agreed the items were describing them, using a 4-point Likert-type scale from 1 (strongly disagree) to 4 (strongly agree). Item scores were summed to give a total score for each subscale (range $=4-40$ ). The higher the subscale score, the higher the importance of the specific face orientation on one's cognitions and behaviors. The PAFO Scale Short Form was validated in a previous study with a Cronbach's alpha of .70 (Wang, 2002). In this study, the PAFO achieved satisfactory internal consistencies, with an alpha value of .75 for the P-subscale and .86 for the A-subscale.

Demographic characteristics. Participants were asked to provide information about their gender, age, year of study, length of the referent intimate relationship (most recent intimate relationship), and cohabitation status in this study. They were also asked whether they had had sex with the referent partner. In addition, four items about participants' parents and family were asked: father's highest education attainment, mother's highest education attainment, parents' marital status, and family income. The socioeconomic status (SES) scale was created to measure socioeconomic status by computing the number of years of education completed by the student's father and mother and family income (Straus, 2004b). The mean score on the SES scale was $8.5(S D=3.8)$. The alpha coefficient of reliability for the SES scale was .70.

Statistical analyses. The prevalence rates of different types of IPV were summarized, and gender differences of the prevalence rates were examined using chi-square tests. To test the association between PFO, AFO, and IPV perpetration, separate logistic regression analyses were employed. The associations between participants' gender, age, SES, and dating violence perpetration were also tested using logistic regressions. In addition, the association between participants' city of residence (i.e., Hong Kong, Shanghai, or Beijing) and IPV was tested given their possible differences in the levels of exposure and openness to Western culture and therefore the influence that traditional Chinese beliefs had on them. Lifetime and preceding-year prevalence of perpetration of different types of IPV was used as dependent variables of the regression models. To test the independent association between each factor and dating violence, each logistic regression model was adjusted 
for other factors. For instance, when testing the association between PFO and lifetime physical violence, PFO was used as the independent variable, lifetime physical violence as the dependent variable, and AFO, city of residence, gender, age, and SES as control variables. Before regression analyses were performed, multicollinearity was checked among all independent and control variables. The variance inflation factor (VIF), which measures how much the variance of a coefficient is increased because of collinearity, was checked and ensured to be no greater than two, which is the general acceptable values for VIF (O'Brien, 2007). In this study, the level of significance was taken as 5\%, and SPSS version 17 was used for the statistical analysis.

\section{Results}

\section{Prevalence of Dating Violence}

Table 2 shows the lifetime prevalence and preceding-year prevalence rates of perpetration of different types of dating partner violence. Psychological aggression was the most common type of violence perpetration reported (lifetime: $71.6 \%$; preceding year: $61.0 \%$ ). Significant gender differences were found in the rates of all types of dating violence perpetration. Specifically, a greater proportion of females than males reported perpetration of physical and psychological violence in an intimate relationship. In contrast, a greater proportion of males admitted perpetration of sexual violence.

\section{Face Orientation and Dating Violence}

Table 3 summarizes the results of the logistic regression analyses testing the associations between dating violence perpetration and different independent variables. When sociodemographic factors (i.e., city of residence, gender, age, and SES) were controlled for, PFO was not significantly associated with the perpetration of any dating violence. After adjusting for other factors, AFO was positively associated with physical and psychological IPV (adjusted odds ratios $[\mathrm{a} O R]=1.043-1.079$; all $p<.001$ ); however, its association with sexual violence perpetration was not statistically significant. Specifically, individuals with higher scores for the A-subscale of the PAFO were more likely than those with lower scores to report perpetration of physical and psychological dating violence, both over their lifetime and during the preceding year.

No significant gender difference was found in the scores for overall face orientation and PFO. However, the AFO scores were significantly higher 
Table 2. Prevalence of Dating Violence Perpetration

\begin{tabular}{|c|c|c|c|c|}
\hline \multirow[b]{2}{*}{ Dating Violence } & \multicolumn{3}{|c|}{ Prevalence (\%) } & \multirow[b]{2}{*}{$\chi^{2}$} \\
\hline & All & Male & Female & \\
\hline \multicolumn{5}{|l|}{ Lifetime } \\
\hline \multicolumn{5}{|l|}{ Physical } \\
\hline Total & 47.7 & 34.2 & 54.3 & $0.000 * * *$ \\
\hline Severe & 22.5 & 16.2 & 25.7 & $0.000 * * *$ \\
\hline \multicolumn{5}{|l|}{ Psychological } \\
\hline Total & 71.6 & 67.3 & 73.8 & $0.005^{* *}$ \\
\hline Severe & 44.8 & 36.4 & 49.1 & $0.000 * * *$ \\
\hline \multicolumn{5}{|l|}{ Sexual } \\
\hline Total & 17.5 & 23.0 & 14.7 & $0.000 * * *$ \\
\hline Severe & 6.3 & 9.4 & 4.7 & $0.000 * * *$ \\
\hline Any kind of violence & 77.3 & 72.1 & 80.0 & $0.000 * * *$ \\
\hline \multicolumn{5}{|l|}{ Preceding-year } \\
\hline \multicolumn{5}{|l|}{ Physical } \\
\hline Total & $4 I .7$ & 29.8 & 47.6 & $0.000 * * *$ \\
\hline Severe & 19.8 & 14.1 & 22.6 & $0.000 * * *$ \\
\hline \multicolumn{5}{|l|}{ Psychological } \\
\hline Total & 61.0 & 52.7 & 65.2 & $0.000 * * *$ \\
\hline Severe & 38.5 & 30.9 & 42.3 & $0.000 * * *$ \\
\hline \multicolumn{5}{|l|}{ Sexual } \\
\hline Total & 14.4 & 18.5 & 12.4 & $0.002 * *$ \\
\hline Severe & 5.7 & 8.8 & 4.1 & $0.000 * * *$ \\
\hline Any kind of violence & 66.3 & 57.1 & 71.0 & $0.000 * * *$ \\
\hline
\end{tabular}

$*_{p}<.05 . *_{p}<.01 . * * * p<.001$.

among male than female $\left(M_{\mathrm{m}}=27.2, S D_{\mathrm{m}}=4.8 ; M_{\mathrm{f}}=26.7, S D_{\mathrm{f}}=4.4 ; t=\right.$ $2.16, p<.05)$.

\section{Other Correlates}

Also shown in Table 3, the sociodemographic factors, including city of residence, gender, age, and SES, were associated with dating violence perpetration. In particular, when compared with participants in Hong Kong, the odds for participants in Shanghai to report perpetration of IPV were about 31.4\% to $69.1 \%$ lower $(\mathrm{aOR}=0.309-0.686$, all $p<.01)$. The odds for participants in Beijing to report IPV perpetration, when compared with those in Hong 


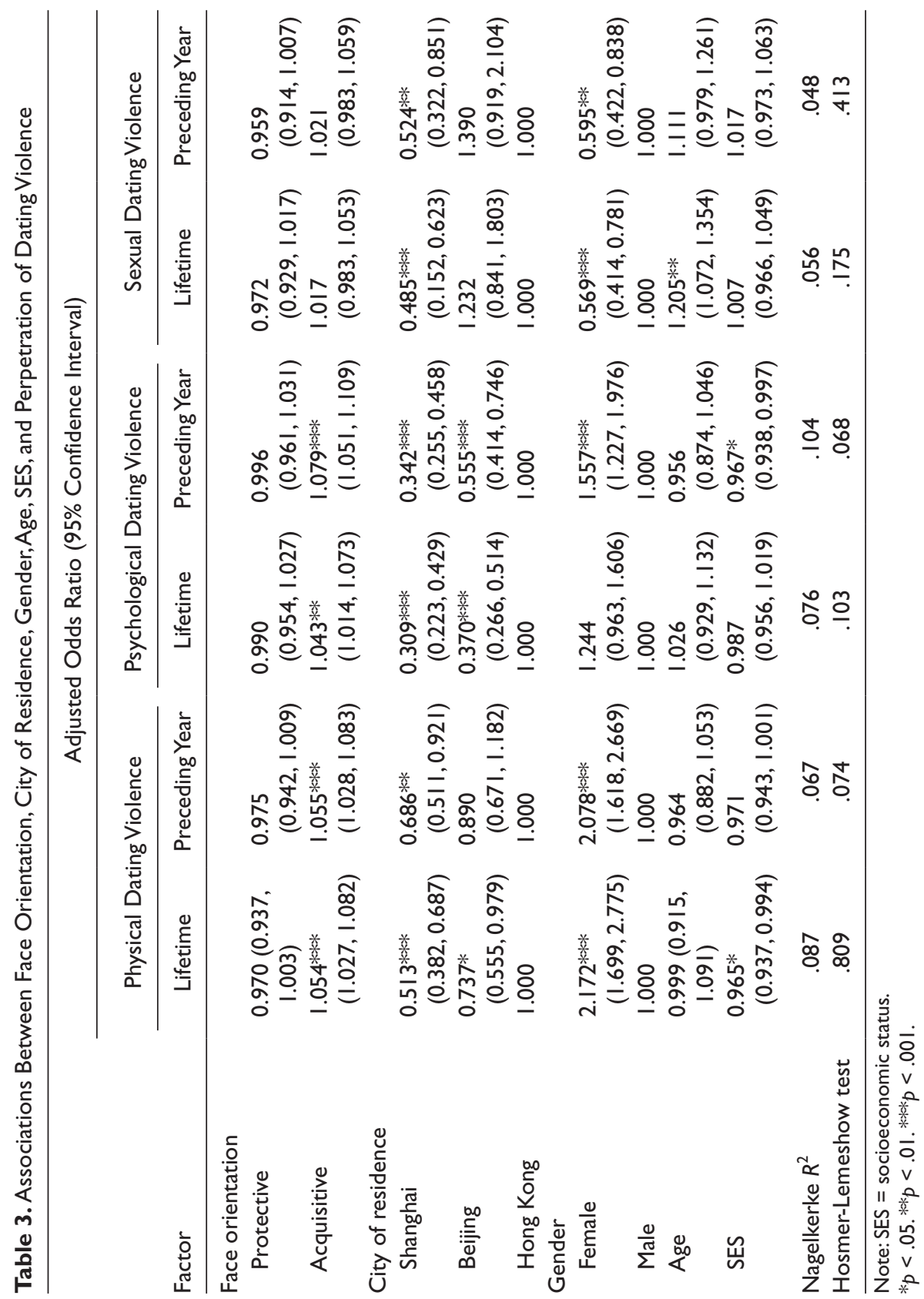


Kong, were $26.3 \%, 63.0 \%$, and $44.5 \%$ lower in lifetime physical dating violence, lifetime psychological violence, and psychological violence in the preceding year, respectively $(\mathrm{a} O R=0.370-0.737$, all $p<.05)$.

Females were more likely than males to report perpetration of physical and psychological violence $(\mathrm{a} O R=1.557-2.172$, all $p<.001)$. However, the reverse was true for sexual violence, for which females were less likely than males to report having used $(\mathrm{aOR}=0.569-0.595$, all $p<.01)$.

The effects of age and SES on the report of dating violence perpetration were less consistent in this study. Age was only statistically associated with sexual violence perpetration over lifetime $(a O R=1.205 ; 95 \%$ confidence interval [CI] [1.072, 1.354], $p<.01)$. SES, however, was negatively associated with physical violence perpetration over lifetime $(a O R=0.965 ; 95 \% \mathrm{CI}$ $[0.937,0.994], p<.05)$ as well as psychological violence perpetration during the preceding year $(a O R=0.967 ; 95 \%$ CI $[0.938,0.997], p<.05)$.

\section{Discussion}

This study aimed at testing the association between the Chinese concept of face orientation and the perpetration of IPV among Chinese populations. Overall, the findings supported the hypothesis that AFO was significantly associated with IPV perpetration, although the findings about PFO were inconclusive. This study also explored the relationship between sociodemographic factors and IPV and found that city of residence, gender, age, and SES could be associated with IPV perpetration.

This study revealed that psychological aggression was the most prevalent type of partner violence perpetration in the three Chinese cities, followed by physical assault and sexual violence. Overall, the prevalence rates found in this study were comparable with existing findings on dating violence among college students (Straus, 2004b), providing evidence supporting that dating violence is prevalent among university students (Straus, 2004b; Sugarman \& Hotaling, 1989).

AFO was positively associated with perpetration of physical and psychological IPV. Being concerned with gaining face, high AFO individuals often crave for recognition and respect, which they may attempt to achieve by all means (Chou, 1996). Oftentimes, their pursuit of self-promotion may appear aggressive to others, especially when they have to devaluate others to promote themselves. In particular, they may even derogate another's face, be pushy toward others, and in extreme cases retaliate against others who denigrate them (Chou, 1996). High AFO individuals may also expect absolute compliance and respect from their partners, which are attributes of face but also a 
demand that is unreasonable and unrealistic (Chan, 2009[AQ: 2]). When their partners fail to fulfill their face needs, they may resolve to use verbal and physical violence to satisfy their needs. Chou (1996) found that high AFO individuals are often perceived as more aggressive in social interactions; however, whether these individuals are more physically aggressive are not yet clear. This study provided supportive evidence for the link between AFO and physical violence perpetration, and future studies may test whether aggression is an underlying factor for the relationship between these two variables.

However, findings in this study did not support the relationship between PFO and perpetration of partner violence. Unlike the present study, the study conducted by Hall et al. (2005) has demonstrated a link between sexual aggression and the loss of face, which is similar to PFO which emphasizes the avoidance of face loss. The lack of consistency in the association between the violence and face loss/fear of face loss calls for more systematic and rigorous studies for further investigation. In particular, there is still the possibility that, even though PFO did not function like loss of face which has been found to be a protective factor against violence (Hall et al., 2005), it may carry a slight buffering effect. All in all, this study should be considered the first of many studies that take into account both types of face orientation in the discussion of partner violence.

The prevalence rates of IPV perpetration differed across cities. With individuals in Hong Kong being the referent group, those in Shanghai and Beijing were less likely to report perpetration of IPV. The differences in prevalence rates may be due to the sociocultural variations between Hong Kong and cities in mainland China. For example, Hong Kong, a city which had been ruled by British colonial government, has been more exposed to Western culture and may therefore be less adhesive to traditional Chinese values. However, residents in mainland China may hold traditional cultural beliefs that could hinder the disclosure of IPV, including fear of criticism and fear of disappointing relatives (Montalvo-Liendo, 2008). Future studies are advised to explore the underlying factors that lead to the differences in prevalence rates across cities in China.

The association between gender and IPV perpetration was supported by the findings in this study. Compared with men, women were found to be more likely to report perpetration of physical and psychological violence but less likely to report perpetration of sexual assault. This pattern of gender asymmetry was comparable with those revealed by previous studies from the United States (Straus, 2004b) and China (Chan \& Straus, 2008; Chan, Straus, et al., 2008). Furthermore, the greater proportion of males who reported perpetration of sexual violence found in this study replicated past findings 
(Anderson \& Savage, 2005; Chan, Brownridge, Leung, Tiwari, \& Ho, 2008; Chan \& Straus, 2008; Jackson, 1999). One possible explanation can be the difference in reporting patterns across gender as shown in previous studies (Dobash \& Dobash, 2004; Stets \& Straus, 1990; Szinovacz \& Egley, 1995).

Gender differences appear not only in the rates of IPV perpetration but also in the levels of AFO. On average, men had a higher level of AFO than women. Given the finding that AFO was positively associated with IPV perpetration, one may expect a higher level of IPV perpetration among men. Yet the opposite was found: Men were less likely to report IPV perpetration. Such finding highlighted a possibility that the associations between gender, AFO, and IPV perpetration were not simply linear. There might be different possible underlying relationships: Gender might interact with AFO or other factors to affect IPV perpetration; it might also serve as a moderating factor for the relationship between AFO and IPV. Future studies may consider investigating the actual patterns of associations among these variables to give a clearer picture of the actual roles of gender and AFO play in affecting IPV perpetration.

Not all types of IPV perpetration were related to age and SES. In this study, older age was associated with increased tendency to report perpetration of sexual partner violence. This finding was inconsistent with other studies (Goodwin et al., 2000; Hedin, 2000; Martin et al., 2001; Muhajarine \& D'Arcy, 1999; Tang, 1999), where younger age was found to be a risk factor for IPV. In contrast, SES, which represented an individual's family income and family education achievement, was negatively associated with physical and psychological IPV. This provided supportive evidence for the claim that higher SES can be a protective factor for partner violence perpetration (Bates et al., 2004; Cunradi et al., 2002).

\section{Limitations}

This study was among the first to explore the associations between PFO, AFO, and perpetration of IPV and was successful in demonstrating the possible link between AFO and perpetration of physical and psychological partner violence; yet it had certain limitations. First, this study used a convenience sample of university students. Thus, the findings cannot be generalized to other cities or populations with younger age or lower education levels. The limited age range, education levels, and even SES may also reduce the generalizability. Future research may consider examine the association between face orientation and IPV more carefully using large, representative sample; and may also conduct comparisons between Chinese and Western populations. 
Second, the study was conducted with a retrospective cross-sectional design. As the real prevalence of IPV is hard to detect, whether the self-reported prevalence rates were accurate was almost impossible to test. There was a possibility that some participants overreported whereas some others underreported. The inclusion of reports from other sources (e.g., official police reports, children's or other family member's reports) may help solve this problem.

Third, the sample in this study comprised $70 \%$ women. It might reflect the actual male-to-female ratio in the universities of the cities; however, it could also be possible that men were less willing to participate. The gender differences in IPV perpetration found in this study might be due to the lower tendency for men to participate and thus to admit perpetration of violence, which might also be related to maintaining face.

Finally, the measures used to assess partner violence may be another confounding factor in the present study. Some researchers have argued that the CTS may have flaws that lead to a finding of gender symmetry in the prevalence of IPV (Giles, 2004; Kimmel, 2002). The most contentious methodological issue with regard to the CTS may be its failure to capture the intent, circumstances, and consequences of violent acts (Giles, 2004; Kimmel, 2002). For example, a woman pushing a man in self-defense and a man intentionally pushing a woman down the stairs would receive the same CTS score. Future studies might employ other measures of violence so as to obtain a more complete profile for the prevalence of IPV perpetration.

\section{Implications}

Despite the above limitations, this study had important implications for future research. First, it highlighted the importance of taking into account the concept of face when exploring factors that are associated with dating partner violence. Nonetheless, this line of research should not be limited to Chinese societies. As Western culture often instills the concept of individualism, it is not surprising to find that AFO is even more prominent and stronger in the West (Ho, 1993). More studies should be carried out to explore whether the concept of face has the same impact on dating violence among Western cultures.

\section{Conclusion}

This study provided preliminary evidence to support the relationship between AFO and IPV perpetration among Chinese populations and gave insights on the possible mechanisms between the concept of face and IPV perpetration for future research to explore. 


\section{Declaration of Conflicting Interests}

The author(s) declared no potential conflicts of interest with respect to the research, authorship, and/or publication of this article.[AQ: 3]

\section{Funding}

The author(s) received no financial support for the research, authorship, and/or publication of this article.[AQ: 4]

\section{References}

Anderson, P. B., \& Savage, J. S. (2005). Social, legal, and institutional context of heterosexual aggression by college women. Trauma, Violence, \& Abuse, 6(2), 130-140.

Balmer, N., Pleasance, P., Buck, A., \& Walker, H. C. (2005). Worried sick: The experience of debt problems and their relationship with health, illness and disability. Social Policy and Society, 5(1), 39-51.

Bates, L. M., Schuler, S. R., Islam, F., \& Islam, M. K. (2004). Socioeconomic factors and processes associated with domestic violence in Rural Bangladesh. International Family Planning Perspectives, 30(4), 190-199.

Caetano, R., McGrath, C., Ramisetty-Mikler, S., \& Field, C. A. (2005). Drinking, alcohol problems and the five-year recurrence and incidence of male to female and female to male partner violence. Alcoholism: Clinical and Experimental Research, 29(1), 98-106.

Carr, M. (1993). Chinese "face" in Japanese and English (Part 2). Review of Liberal Arts, 85, 69-101.

Campbell, J. C., \& Soeken, K. L. (1999). Forced sex and intimate partner violence: Effects on women's risk and women's health. Violence Against Women, 5, 1017-1035.

Chan, K. L. (2006). The Chinese concept of face and violence against women. International Social Network, 49(1), 65-73.

Chan, K. L. (2007). Sexual violence against women and children in China. Pretoria, South Africa: Sexual Violence Research Initiative.

Chan, K. L., Brownridge, D. A., Leung, W. C., Tiwari, A., \& Ho, H. W. Y. (2008). Mental health profile of sexual violence perpetrators among university students in Hong Kong. In J. K. Quinn \& I. G. Zambinim (Eds.), Family relations: 21st century issues and challenges (pp. 57-70). Hauppauge, NY: Nova Science.

Chan, K. L., Brownridge, D. A., Tiwari, A., Fong, D. Y. T., \& Leung, W. C. (2008). Understanding violence against Chinese women in Hong Kong: An analysis of risk factors with a special emphasis on the role of in-law conflict. Violence Against Women, 14, 1295-1312.

Chan, K. L., \& Straus, M. A. (2008). Prevalence and correlates of physical assault on dating partners. Open Social Science Journal, 1, 5-14. 
Chan, K. L., Straus, M. A., Brownridge, D. A., Tiwari, A., \& Leung, W. C. (2008). Prevalence of dating partner violence and suicidal ideation among male and female university students worldwide. Journal of Midwifery \& Womens Health, 53, 529-537.

Chou, M. L. (1996). Protective and acquisitive face orientation: A person by situation approach to face dynamic in social interaction (Doctoral dissertation). The University of Hong Kong, Hong Kong, China.

Cunradi, C. B., Caetano, R., \& Schafer, J. (2002). Socioeconomic predictors of intimate partner violence among White, Black, and Hispanic couples in the United States. Journal of Family Violence, 17, 377-389.

Dobash, R. P., \& Dobash, R. E. (2004). Women's violence to men in intimate relationships: Working on a puzzle. British Journal of Criminology, 44, 324-349.

Eberhard, W. (1967). Guilt and sin in traditional China. Berkeley: University of California Press.

Giles, J. R. (2004). Growing through adversity: Becoming women who live without partner abuse. A grounded theory study. Auckland, New Zealand: School of Psychotherapy, Auckland University of Technology.

Goffman, E. (1955). On face-work: An analysis of ritual elements in social interaction. Psychiatry, 18, 213-231.

Golinelli, D., Longshore, D., \& Wenzel, S. L. (2009). Substance use and intimate partner violence: Clarifying the relevance of women's use and partners' use. Journal of Behavioral Health Services \& Research, 36(2), 199-211.

Goodwin, M. M., Gazmararian, J. A., Johnson, C. H., Gilbert, B. C., \& Saltzman, L. E. (2000). Pregnancy intendedness and physical abuse around the time of pregnancy: Findings from the pregnancy risk assessment monitoring system, 19961997. PRAMS Working Group. Pregnancy Risk Assessment Monitoring System. Maternal and Child Health Journal, 4(2), 85-92.

Hall, G. C. N., Teten, A. L., DeGarmo, D. S., Sue, S., \& Stephens, K. A. (2005). Ethnicity, culture, and sexual aggression: Risk and protective factors. Journal of Consulting and Clinical Psychology, 73, 830-840.

Hedin, L. W. (2000). Postpartum, also a risk period for domestic violence. European Journal of Obstetrics, Gynecology, and Reproductive Biology, 89(1), 41-45.

Ho, D. Y. F. (1976). On the concept of face. American Journal of Sociology, 81, 867-884.

Ho, D. Y. F. (1980). Face and stereotyped notions about Chinese face behavior. Philippine Journal of Psychology, 13(1\&2), 20-33.

Ho, D. Y. F. (1993). Face dynamics: From conceptualization to measurement. In S. Ting-toomey (Ed.), The challenge of facebwork (pp. 269-286). New York, NY: SUNY Press.

Hu, H. C. (1944). The Chinese concepts of face. American Anthropologist, 46, 45-64.

Huang, S. (1987). Two studies of prototype semantics: Xiao "filial piety" and mei mianzi "loss of face." Journal of Chinese Linguistics, 15, 55-89. 
Jackson, S. M. (1999). Issues in the dating violence research: A review of the literature. Aggression \& Violent Behaviour, 4, 233-247.

Kimmel, M. S. (2002). "Gender symmetry" in domestic violence: A substantive and methodological research review. Violence Against Women. Special Issue: Women's Use of Violence in Intimate Relationships Part I, 8, 1332-1363.

King, A. Y. C., \& Myers, J. T. (1977). Shame as an incomplete concept of Chinese culture: A study of face. Hong Kong: The Chinese University of Hong Kong Social Research Centre.

Lau, Y., \& Wong, D. F. K. (2008). Are concern for face and willingness to seek help correlated to early postnatal depressive symptoms among Hong Kong Chinese women? A cross-sectional questionnaire survey. International Journal of Nursing Studies, 45(1), 51-64.

Lehrer, J. A., Buka, S., Gortmaker, S., \& Shrier, L. A. (2006). Depressive symptomatology as a predictor of exposure to intimate partner violence among US female adolescents and young adults. Archives of Pediatrics and Adolescent Medicine, 160, 270-276.

Lindhorst, T., \& Oxford, M. (2008). The long-term effects of intimate partner violence on adolescent mothers' depressive symptoms. Social Science \& Medicine, 66, 1322-1333.

Martin, S. L., Mackie, L., Kupper, L. L., Buescher, P. A., \& Moracco, K. E. (2001). Physical abuse of women before, during, and after pregnancy. Journal of the American Medical Association, 285, 1581-1584.

Montalvo-Liendo, N. (2008). Cross-cultural factors in disclosure of intimate partner violence: An integrated review. Journal of Advanced Nursing, 65, 20-34.

Muhajarine, N., \& D’Arcy, C. (1999). Physical abuse during pregnancy: Prevalence and risk factors. CMAJ: Canadian Medical Association Journal, 160, 1007-1011.

O'Brien, R. M. (2007). A caution regarding rules of thumb for variance inflation factors. Quality and Quantity, 41, 673-690.

Stets, J. E., \& Straus, M. A. (1990). Gender differences in reporting marital violence and its medical and psychological consequences. In M. A. Straus \& R. Gelles (Eds.), Physical violence in American families. London, UK: Transaction.[AQ: 5]

Straus, M. A. (2004a). Cross-cultural reliability and validity of the revised Conflict Tactics Scales: A study of university student dating couples in 17 nations. CrossCultural Research, 38, 407-432.

Straus, M. A. (2004b). Prevalence of violence against datnig partners by male and female university students worldwide. Violence Against Women, 10, 790-811.

Straus, M. A., Hamby, S. L., BoneyMcCoy, S., \& Sugarman, D. B. (1996). The revised Conflict Tactics Scales (CTS2)_-Development and preliminary psychometric data. Journal of Family Issues, 17, 283-316. 
Stuart, G. L., \& Holtzworth-Munroe, A. (2005). Testing a theoretical model of the relationship between impulsivity, mediating variables, and husband violence. Journal of Family Violence, 20, 291-303.

Sugarman, D. B., Aldarondo, E., \& Boney-McCoy, S. (1996). Risk marker analysis of husband-to-wife violence: A continuum of aggression. Journal of Applied Social Psychology, 26, 313-337.

Sugarman, D. B., \& Hotaling, G. T. (1989). Dating violence: Prevalence, context, and risk markers. In A. A. P.-G. J. E. Stets (Ed.), Violence in dating relationships: Emerging social issues (pp. 3-31). New York, NY: Praeger.

Szinovacz, M. E., \& Egley, L. C. (1995). Comparing one-partner and couple data on sensitive marital behaviors: The case of marital violence. Journal of Marriage \& the Family, 57, 995-1010.

Tang, C. S. K. (1999). Wife abuse in Hong Kong Chinese families: A community survey. Journal of Family Violence, 14(2), 173-191.

Tiwari, A., Wong, J., Brownridge, D. A., Chan, K. L., Fong, D. Y. T., Leung, W. C., \& Ho, P. C. (2009). Psychological intimate partner abuse among Chinese women: What we know and what we still need to know. Open Social Science Journal, 2, 32-36.

Vives-Cases, C., Gil-Gonzalez, D., \& Carrasco-Portino, M. (2009). Verbal marital conflict and male domination in the family as risk factors of intimate partner violence. Trauma, Violence, \& Abuse, 10, 171-180.

Wang, H. (2002). Help seeking tendency in situation of threat to self-esteem and facelosing. Hong Kong, China: University of Hong Kong Press.

Whitaker, D. J., \& Reese, L. (2007). Preventing intimate partner violence and sexual violence in racial/ethnic minority communities: CDC's demonstration projects. Atlanta, GA: National Center for Injury Prevention and Control, Centers for Disease Control and Prevention.

Yoshihama, M., Horrocks, J., \& Kamano, S. (2009). The role of emotional abuse in intimate partner violence and health among women in Yokohama, Japan. American Journal of Public Health, 99, 647-653.

\section{Bio}

Ko Ling Chan is with the Department of Social Work and Social Administration at the University of Hong Kong, Hong Kong. 